\title{
Numerical and analytical approach for the spin-stabilized satellite attitude propagation
}

\author{
M. C. Zanardi ${ }^{1}$ - V. Orlando ${ }^{2}$. G. B. Motta ${ }^{3}$. \\ T. Pelosi ${ }^{3}$ - W. R. Silva ${ }^{3,4}$
}

Received: 30 April 2015 / Revised: 11 February 2016 / Accepted: 27 February 2016 /

Published online: 31 March 2016

(c) SBMAC - Sociedade Brasileira de Matemática Aplicada e Computacional 2016

\begin{abstract}
This paper presents the comparison between the numerical and analytical results of a spacecraft attitude propagation for a spin-stabilized satellite. Some external torques are introduced in the equations of the motion and the comparisons are done considering that these torques are acting together, which are: gravity gradient, aerodynamic, solar radiation, magnetic residual and eddy current. In the numerical approach it is used the quaternion to represent the attitude. This numerical approach can be applied for any kind of satellite. The analytical approach is applied directly for a spin-stabilized satellite and the equations of motion are described in terms of the spin velocity, spin axis right ascension and declination angles. An analytical solution of these equations is presented and valid for one orbit period. Applications are developed considering the Brazilian spin-stabilized satellites SCD1 and SCD2. The comparisons are important to validate some simplifications that are required in
\end{abstract}

Communicated by Elbert E. N. Macau, Antônio Fernando Bertachini de Almeida Prado and Cristiano Fiorilo de Melo.

M. C. Zanardi

mceciliazanardi@gmail.com

V. Orlando

valcir@ccs.inpe.br

G. B. Motta

gabriel_borderes@yahoo.com.br

T. Pelosi

tatiane.pelosi@gmail.com

W. R. Silva

reis.william@gmail.com

1 UFABC-Federal University of ABC, Santo André, SP CEP 09210-180, Brazil

2 Space Mechanics and Control Division, Brazilian Institute for Space Research (INPE) São Paulo, São José dos Campos CEP 12227-010, Brazil

3 UNESP-São Paulo State University, Guaratinguetá, SP CEP 12516-410, Brazil

4 ITA-Technological Institute of Aeronautics São Paulo, São José dos Campos CEP 12228-900, Brazil 
the analytical approach. The results show that the average components of the external torque are sufficient to observe the main influence of the considered torques.

Keywords Attitude prediction · Quaternion · Euler angles · External torques · Spin axis

Mathematics Subject Classification 37N05

\section{Introduction}

The goal of this paper is to compare the numerical and analytical results of a spacecraft attitude propagation. Some external torques are introduced in the equations of the motion and the comparisons are done considering all torques acting together. The considered torques are: gravity gradient, aerodynamic, solar radiation and magnetic torques.

In the numerical approach it is used the quaternion to represent the attitude and the 8th order Runge-Kutta method to integrate the equation of motion. The dynamic equations of the satellite's rotational motion are described by the Euler equations and the four kinematic equations for the attitude quaternion. The simulations are developed in FORTRAN language.

Applications are developed considering the Brazilian spin-stabilized satellites SCD1 and SCD2, which are quite appropriated for verification and comparison of the theory with the real data acquired and processed by the Satellite Control Center of National Institute Space Research from Brazil (SCC/INPE). The operations and initial behaviors of the SCD1 and SCD2 are discussed in the studies by Carrara and Guedes (1994), Kuga et al. (1999) and Orlando et al. (1997). Spin-stabilized satellites have the spin axis along the biggest principal moment of inertia's axis. A spherical coordinate system fixed in the satellite is used to locate the satellite spin axis in relation to the terrestrial equatorial system. The spin axis direction is specified by its right ascension and the declination angles and is represented in Fig. 1. The time evolution of these angles is gotten from the numerical results of the quaternion attitude propagation.

The analytical approach is applied directly for a spin-stabilized satellite and the equations of motion are described in terms of the spin velocity and the spin axis right ascension and declination angles. The developments of these equations are similar to those reported by Kuga et al. (1987a) and Kuga et al. (1987b). In these references are considered the magnetic torques and the gravity gradient torques and the numerical applications are done for spin-stabilized satellite. In this paper, the averages of the components of each torque over an orbital period

Fig. 1 Equatorial system $(\hat{\mathrm{I}}, \hat{\mathrm{J}}, \hat{\mathrm{K}})$, spin axis orientation $(\hat{\mathrm{k}})$, right ascension $(\alpha)$ and declination $(\delta)$ of the spin axis.

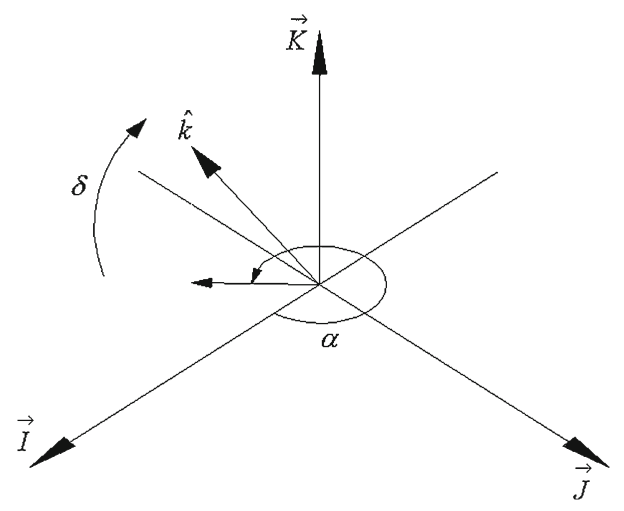


are used to get an analytical solution of these equations. The numerical implementation in this case is done with software MATLAB.

The comparisons are important to validate some simplifications that were required in the analytical approach.

\section{Considerations about external torques}

Classical models (Pisacane and Moore 1994; Wertz 1978) are assumed for each considered torque in terms of the quaternion parametrization for the numerical approach and in terms of the right ascension and declination of the spin axis for the analytical approach. In the study by Shrivastava and Modi (1993) is also done a review of environmental forces and their effects in satellite attitude.

The gravity gradient torque is generated by the difference of the Earth gravity force direction and intensity actuating on each satellite mass element. This torque depends on the principal moments of inertia of the satellite and is inversely proportional to the cube of the satellite geocentric distance. The adopted mathematical model in terms of the quaternion attitude is given in (Zanardi et al. 2012) and for the analytical approach is given in (Motta and Zanardi 2014; Zanardi et al. 2014).

The aerodynamic torque is created by the interactions of rarefied air particles with the satellite surface and it has the predominant orbit perturbation effect in LEO orbit satellites. This torque depends on the atmospheric density, the distance between the centre of pressure and the mass centre of the satellite, the magnitude of the satellite's velocity relative to the atmosphere, reference section area of the satellite and the aerodynamic coefficient. In this paper, only the influence of the Drag force is considered for both approach. To estimate the influence of the aerodynamic torque in the rotational motion, in this paper it is assumed that the velocity is equal to the orbit velocity, the aerodynamic coefficient is fixed, and the thermosphere model TD-88 is used for the atmospheric density (Pisacane and Moore 1994). The adopted mathematical model for the analytical approach is given in (Motta and Zanardi 2014; Zanardi et al. 2014) and for the numerical approach in terms of the quaternion is given in (Zanardi et al. 2012).

Magnetic disturbance torques result from the interaction between the spacecraft's residual magnetic field, the Earth's magnetic field and the eddy current. In this paper, it is assumed that the spacecraft is manufactured from material such that the primary sources of magnetic torques are the spacecraft magnetic moments and eddy currents, with other sources neglected.

The residual magnetic torque results from the interaction between the spacecraft's residual magnetic moment and the Earth magnetic field, and its main effect is to produce a spin axis orientation drift. It depends on the residual magnetic moment of the spacecraft and the geocentric magnetic field. In this paper the dipole model is assumed for the Earth's magnetic field (Garcia et al. 2009) and the satellite is supposed to be in an elliptical orbit. The adopted mathematical model for this torque in terms of the quaternion attitude is given in (Zanardi et al. 2014) and in terms of right ascension and declination is given in (Garcia et al. 2009; Motta and Zanardi 2014; Zanardi et al. 2014).

The torque induced by eddy currents is caused by the spacecraft spin motion. It is known (Kuga et al. 1987a; Motta and Zanardi 2014; Shrivastava and Modi 1993) that the eddy currents produce a torque which causes the precession in the spin axis and causes an exponential decay of the spin rate. It depends on the spacecraft's angular velocity vector, the geomagnetic field and of the constant coefficient (which depends on the spacecraft geometry and conductivity).The adopted mathematical model for the analytical approach is given in (Zanardi et al. 
2014), and for the numerical approach, in terms of the quaternion, is given in (Zanardi et al. 2012).

The solar radiation pressure is created by the continuous photons collisions with the satellite surface, which can be able to absorb or reflect on this flow. The total change of the momentum of all the incident photons on the satellite surface originates from the solar radiation force and it can produce a torque. A Solar Radiation Torque model was developed in (Zanardi et al. 1999) for the case which the illuminated surfaces of the satellite are a circular flat surface and a portion of the cylindrical surface. It depends on the solar parameter, the Sun-Earth distance, the satellite geocentric distance, the Sun satellite distance, the specular and total reflection coefficients. For the analytical approach the model for this torque is presented in (Motta and Zanardi 2014) and for the numerical approach it is presented in (Zanardi et al. 2014). In the analytical approach only the mean components of each torque are considered over one orbit period. To obtain the mean torques, it is necessary to integrate the instantaneous torques over one orbital period. These procedures are discussed in (Zanardi et al. 2014), where it is also possible to get the mean components of each torque.

\section{Numerical approach}

For the numerical approach, the dynamic equations of the satellite's rotational motion are described by the Euler equations and the kinematic equations for the attitude quaternion. The Euler equations give the tax of variation of the components of the satellite's spin velocity and depend on the components of the external torques in the body system (satellite fixed system) (Pisacane and Moore 1994; Wertz 1978):

$$
\begin{aligned}
& \dot{p}=q r\left(I_{y}-I_{z}\right) / I_{x}+N_{x} / I_{x}, \\
& \dot{q}=p r\left(I_{z}-I_{x}\right) / I_{y}+N_{y} / I_{y}, \\
& \dot{r}=p q\left(I_{x}-I_{y}\right) / I_{z}+N_{z} / I_{z} .
\end{aligned}
$$

In these equations $I_{x}, I_{y}, I_{z}$ are the Principal Moments of Inertia of the satellite, $p, q, r$ and $N_{x}, N_{y}, N_{z}$ are the components of the spin velocity and the external torques in the body system, respectively.

Different developments of the Euler equations are showed by Mitchell and Richardson (2001), using linear transformations and an arbitrary torque.

In this paper, the kinematic equations are described in terms of the attitude quaternion $q$, which is a $4 \times 1$ vector given by (Pisacane and Moore 1994; Wertz 1978):

$$
q=\left[\begin{array}{llll}
q_{1} & q_{2} & q_{3} & q_{4}
\end{array}\right]^{t}=\left[\begin{array}{ll}
\vec{q} & q_{4}
\end{array}\right]^{t},
$$

where t represents the transposed of the matrix and they can be expressed in function of the rotation angle $(\phi)$ and of the axis of rotation $\vec{n}$ :

$$
\vec{q}=\left[\begin{array}{lll}
q_{1} & q_{2} & q_{3}
\end{array}\right]^{t}=\sin (\phi / 2) \vec{n} \text { and } q_{4}=\cos (\phi / 2) .
$$

The matrix of attitude in terms of the quaternion is presented in Pisacane and Moore (1994). The kinematic equations that describe the tax of variation of the components of the quaternion of attitude, due to rotation of the satellite, are given by (Pisacane and Moore 1994; Wertz 1978):

$$
\begin{array}{llrl}
\dot{q}_{1} & =\frac{1}{2}\left[p q_{4}-q q_{3}+r q_{2}\right], & \dot{q}_{2}=\frac{1}{2}\left[q q_{4}-r q_{1}+p q_{3}\right], \\
\dot{q}_{3}=\frac{1}{2}\left[r q_{4}-p q_{2}+q q_{1}\right], & \dot{q}_{4}=-\frac{1}{2}\left[p q_{1}+q q_{2}+r q_{3}\right] .
\end{array}
$$


The applications are developed for spin-stabilized satellite which has the spin axis along the biggest principal moment of inertia's axis. The direction of the spin axis $\hat{k}$ is specified by its right ascension $(\alpha)$ and the declination $(\delta)$, which are represented in Fig. 1.This spherical coordinate can be obtained using the attitude quaternion $q$ and the components of the spin velocity $W$. If the units vectors $(\hat{I}, \hat{J}, \hat{K})$ are associated with the equatorial system and the units vectors $(\hat{i}, \hat{j}, \hat{k})$ with the body system, then the spin velocity vector can be expressed by:

$$
\vec{W}_{I}=P \hat{I}+Q \hat{J}+R \hat{K} \text { and } \vec{W}=p \hat{i}+q \hat{j}+r \hat{k}
$$

If the components of the attitude quaternion $\left(q_{1}, q_{2}, q_{3}, q_{4}\right)$ and components of the satellite spin velocity $(p, q, r)$ are known, the vectors $\vec{W}$ and $\vec{W}_{I}$ are related by (6):

$$
\begin{aligned}
& P=\left(q_{1}^{2}-q_{2}^{2}-q_{3}^{2}+q_{4}^{2}\right) p+2 q\left(q_{2} q_{1}-q_{4} q_{3}\right)+2 r\left(q_{3} q_{1}+q_{4} q_{2}\right) \\
& Q=2 p\left(q_{1} q_{2}+q_{4} q_{3}\right)+\left(-q_{1}^{2}+q_{2}^{2}-q_{3}^{2}+q_{4}^{2}\right) q+2 r\left(q_{3} q_{2}-q_{4} q_{1}\right) \\
& R=2 p\left(q_{3} q_{1}-q_{4} q_{2}\right)+2 q\left(q_{3} q_{2}+q_{4} q_{1}\right)+\left(-q_{1}^{2}-q_{2}^{2}+q_{3}^{2}+q_{4}^{2}\right) r
\end{aligned}
$$

and the magnitude of the spin velocity is given by:

$$
W=\left(p^{2}+q^{2}+r^{2}\right)^{\frac{1}{2}}
$$

According to Fig. 1, the components of spin velocity P. Q, R can be obtained by:

$$
P=W \cos \delta \cos \alpha, \quad Q=W \cos \delta \sin \alpha, \quad R=W \sin \delta
$$

By the Eq. (8), the right ascension $(\alpha)$ and declination $(\delta)$ of the spin velocity can be computed by:

$$
\sin \delta=\frac{R}{W}, \quad \cos \propto=\frac{p}{W \cos \delta}, \quad \sin \alpha=\frac{Q}{W \cos \delta}
$$

with $0 \leq \delta \leq 90^{\circ}$ and $0 \leq \alpha \leq 360^{\circ}$

Then to compare the numerical and analytical results, the right ascension and declination of the spin velocity will be computed using the numerical results obtained for components of the spin velocity and attitude quaternion by the numerical integration of the Eqs. (1) and (4).

\section{Analytical approach}

For the analytical approach, the motion for spin-stabilized artificial satellites is described by the variations of the spin velocity, the declination and the right ascension of the spin axis and are given by the Euler equations in spherical coordinates (Kuga et al. 1987a):

$$
\frac{d W}{d t}=\frac{1}{I_{z}} N_{z}, \frac{d \delta}{d t}=\frac{1}{I_{z} W} N_{y}, \frac{d \alpha}{d t}=\frac{1}{I_{z} W \operatorname{Cos} \delta} N_{x},
$$

where $I_{z}$ is the moment of inertia along the spin axis, $N_{x}, N_{y}, N_{z}$ are the components of the external torques in the body system and here are given by the sum of the mean components of gravity gradient, aerodynamic, solar radiation, residual magnetic and eddy current torques.

The analytical approach for this case is similar to that of Thomas and Cappelare (1964) and $\mathrm{Yu}$ (1963). Applications in these references were done for the Telstar satellites using the magnetics torque with dipolo model for the geomagnetic field. 
The solution of these equations was gotten in Motta and Zanardi (2014), and for one orbital period they are given by:

$$
\begin{gathered}
W=\left(W_{0}+\frac{N_{\mathrm{gzm}}}{N_{\mathrm{izm}}}\right) e^{\frac{N_{\mathrm{izm}}}{I_{z}} t}-\frac{N_{\mathrm{gzm}}}{N_{\mathrm{izm}}} \\
\delta=\frac{t}{I_{z}}\left(N_{\mathrm{iym}}-\frac{N_{\mathrm{tym}} N_{\mathrm{izm}}}{N_{\mathrm{gzm}}}\right)+\frac{N_{\mathrm{tym}}}{N_{\mathrm{gzm}}} \ln \left(\frac{W}{W_{0}}\right)+\delta_{0}, \\
\alpha=\frac{t}{I_{z} \cos \bar{\delta}}\left(N_{\mathrm{ixm}}-\frac{N_{\mathrm{txm}} N_{\mathrm{izm}}}{N_{\mathrm{gzm}}}\right)+\frac{N_{\mathrm{txm}}}{N_{\mathrm{gzm}} \cos \bar{\delta}} \ln \left(\frac{W}{W_{0}}\right)+\alpha_{0},
\end{gathered}
$$

where $N_{\text {ixm }}, N_{\text {iym }}, N_{\text {izm }}$ are the mean components of eddy currents torques and $N_{\text {tym }}=$ $N_{\text {Aym }}+N_{\text {sym }}+N_{\text {gym }}+N_{\text {rym }}$ and $N_{\text {txm }}=N_{\text {Axm }}+N_{\text {sxm }}+N_{\text {gxm }}+N_{\text {rxm }}$, being $N_{\text {Axm }}, N_{\text {Aym }}, N_{\text {Azm }}, N_{\text {sxm }}, N_{\text {sym }}, N_{\text {szm }}, N_{\text {gxm }}, N_{\text {gym }}, N_{\text {gzm }}, N_{\text {rxm }}, N_{\text {rym }}, N_{\text {rzm }}$ the mean components of the aerodynamic, solar radiation, gravity gradient and residual magnetic torques, respectively, and $\bar{\delta}=\frac{\delta-\delta_{0}}{2}, \delta$ is the computed declination, $W_{0}, \delta_{0}$ and $\alpha_{0}$ are the initial values for spin velocity, declination and right ascension of the spin axis.

The numerical implementation of these analytical solution is developed for the real data of Brazilian satellite SCD1 and SCD2 to compare with the numerical results of Eqs. (1), (4), (7) and (5).

\section{Numerical simulation and results comparison}

The numerical and analytical approaches have been applied to the spin-stabilized Brazilian Satellite (SCD1 and SCD2) for verification and comparison of the approaches against data generated by the SCC/INPE.

The 8th Runge-Kutta method is used to determine the numerical solution for Eqs. (1) and 4 . The numerical solutions give the components of the attitude quaternion and the spin velocity, which are used to compute the spin velocity, right ascension and declination of the

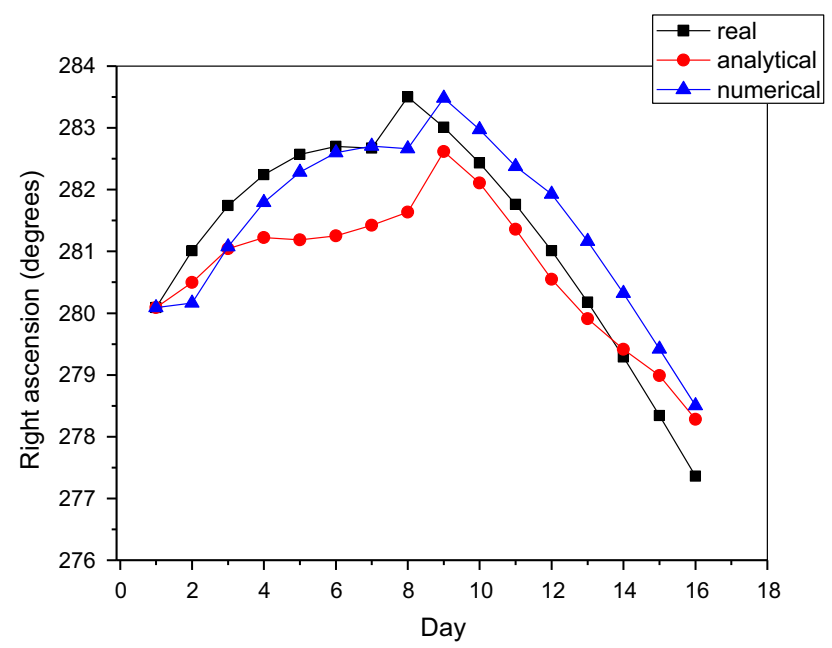

Fig. 2 Temporal variation for the actual, analytical and numerical right ascension of the spin axis for SCD1, with the daily updated data 


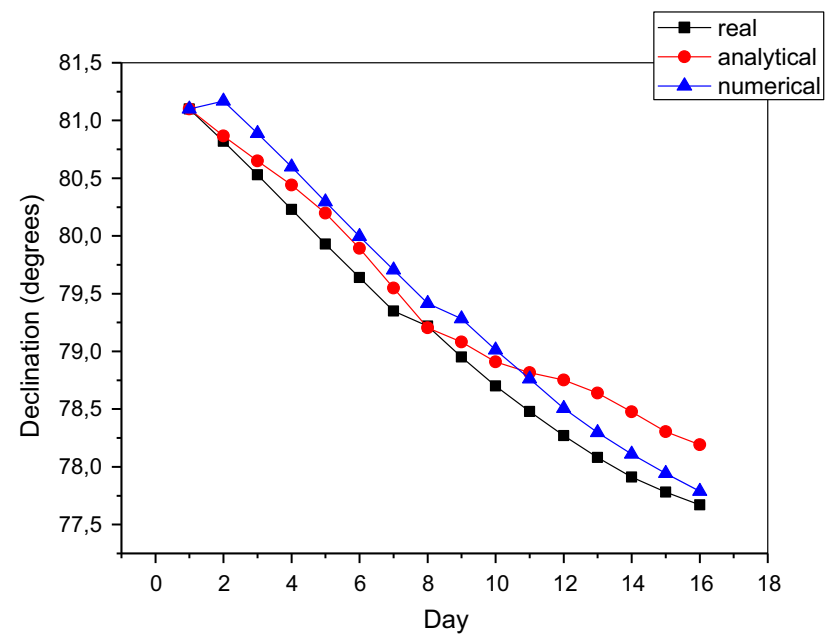

Fig. 3 Temporal variation for the actual, analytical and numerical declination of the spin axis for SCD1, with the daily updated data

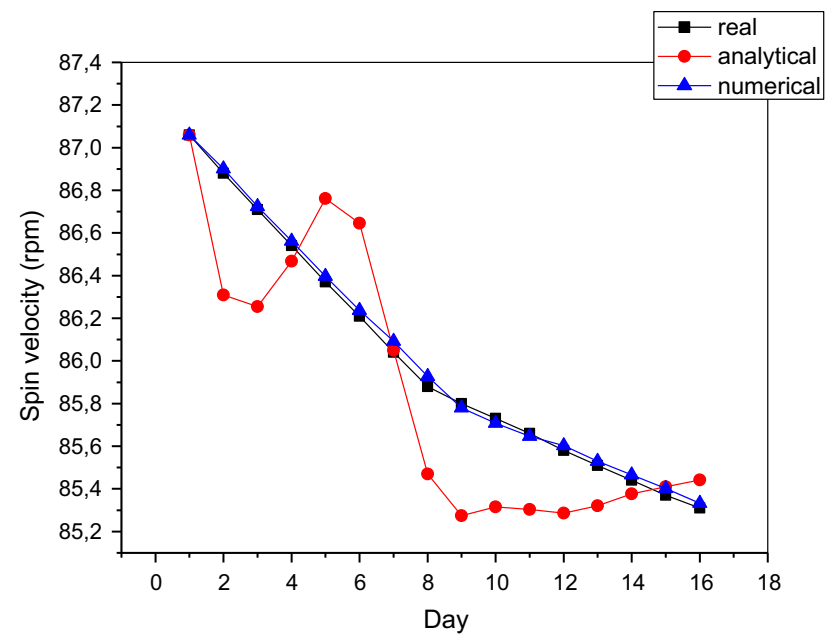

Fig. 4 Temporal variation for the actual, analytical and numerical spin velocity for SCD1 and with the daily updated data

spin axis using Eqs. (7) and (9). Then these numerical values are compared with the numerical values gotten by the analytical solution, given by Eqs. (11)-(13), and with real data supplied by SCC/INPE to check the precision of the presented approaches.

In the simulations, the propagated (analytical and numerical) attitude is daily updated with the actual satellite data and the orbital elements are updated, taking into account the main influences of the Earth oblateness.

Initial conditions for the attitude were taken from CSS/ INPE supplied data (Carrara and Guedes 1994; Kuga et al. 1999). The simulations were made for 16 days for each satellite. It is assumed that the s range of INPE is $0.5^{\circ}$ for the angles and $0.5 \mathrm{rpm}$ for the spin velocity. 


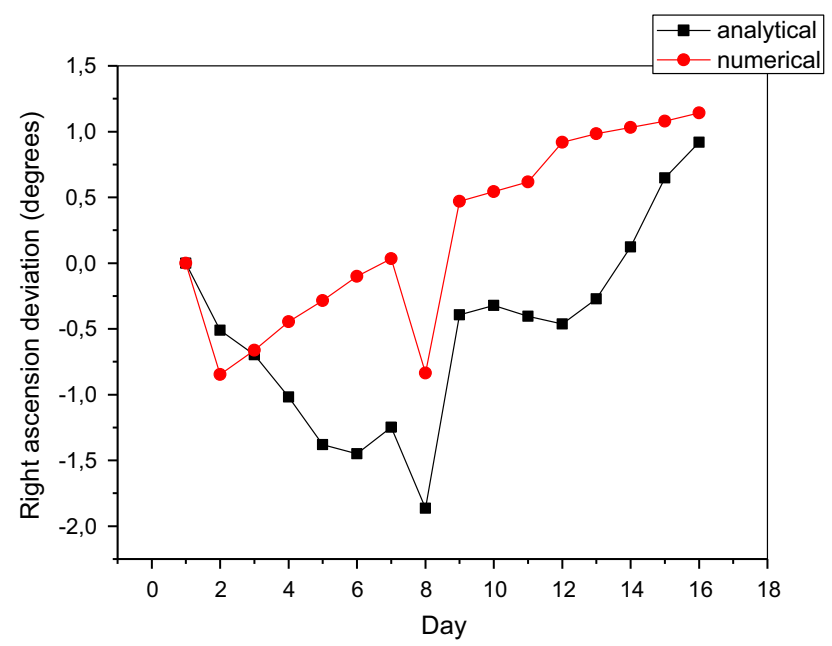

Fig. 5 Temporal variation for the deviation between the analytical or numerical and actual right ascension of the spin axis for SCD1, with the daily updated data

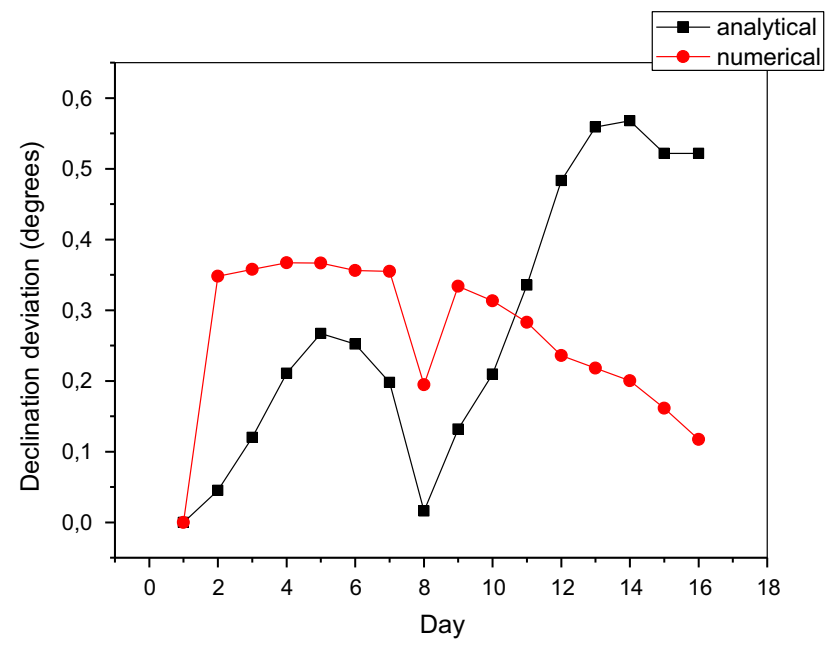

Fig. 6 Temporal variation for the deviation between the analytical or numerical and actual declination of the spin axis for SCD1, with the daily updated data

\section{Applications for SCD1}

For SCD1, the initial conditions of attitude had been taken for date of August, 17, 1993 to the 00:00:00 GMT. In this case, the temporal variations of the right ascension, declination and spin velocity are shown in Figs. 2, 3, 4. In the Figs. 5, 6, 7 are shown the results for the deviation error between the computed values and actual values of the right ascension, declination and spin velocity. In Table 1 are shown the mean deviation errors of each parameter for this simulation.

For this test period, the results showed that mean deviation error in right ascension, declination and spin velocity are within the dispersion range of the attitude determination system 


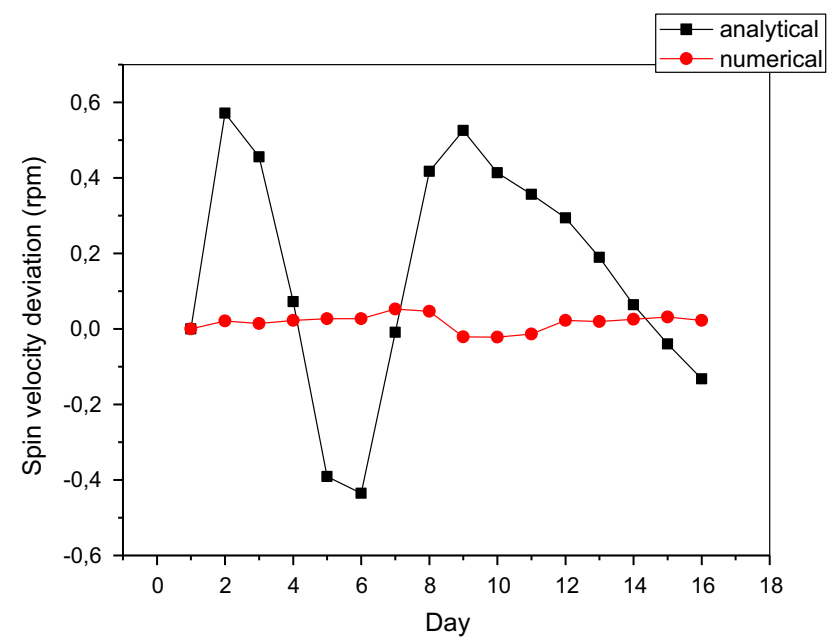

Fig. 7 Temporal variation for the deviation between the analytical or numerical and actual spin velocity for SCD1, with the daily updated data

Table 1 Mean deviation errors for SCD1 simulations with daily updated data

\begin{tabular}{llc}
\hline & Numerical & Analytical \\
\hline Right ascension deviation (degrees) & 0.2652 & -0.5206 \\
Declination deviation (degrees) & 0.2629 & 0.2757 \\
Spin velocity deviation (rpm) & 0.0174 & 0.1471 \\
\hline
\end{tabular}

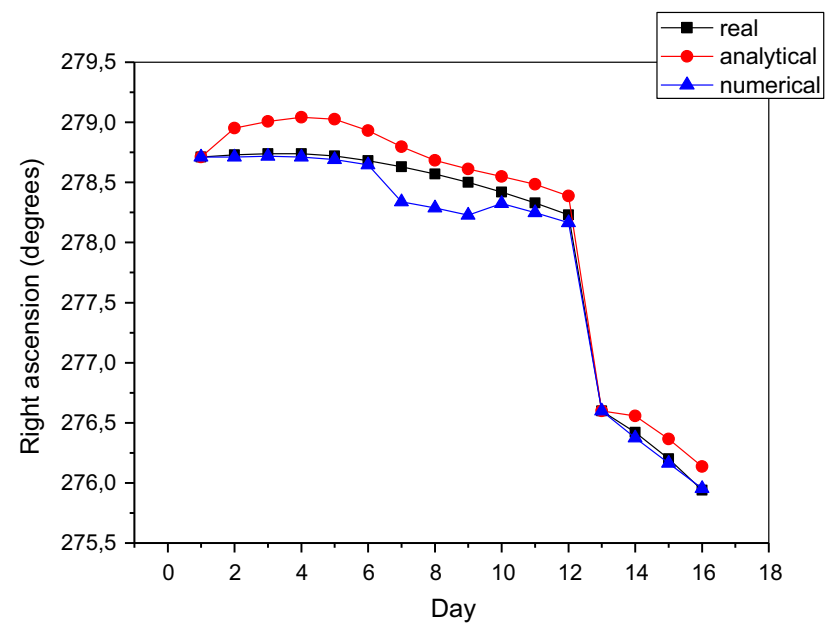

Fig. 8 Temporal variation for the actual, analytical and numerical right ascension of the spin axis for SCD2, with daily updated data 


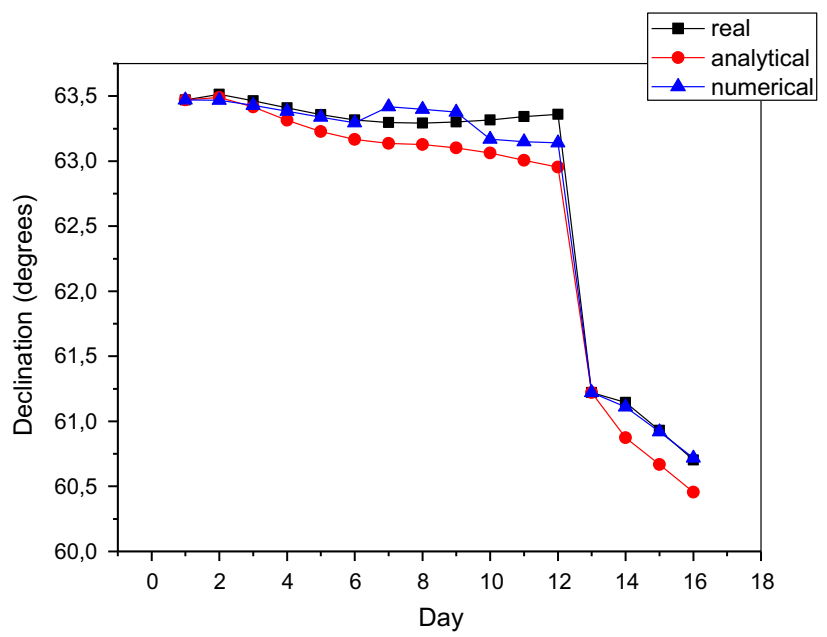

Fig. 9 Temporal variation for the actual, analytical and numerical declination of the spin axis for SCD2, with daily updated data

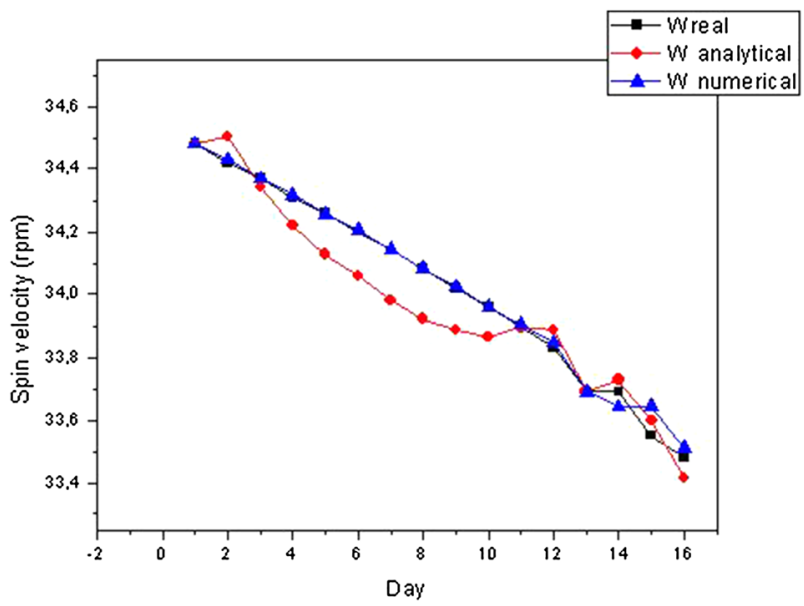

Fig. 10 Temporal variation for the actual, analytical and numerical spin velocity for SCD2, with daily updated data

performance of CSS/INPE. In Table 1 it is possible to observe that the numerical results are better than the analytical approach.

\section{Applications of the SCD2}

For the SCD2, the initial conditions of attitude had been taken for date of February, 12, 2002 to the 00:00:00 GMT. In this case, the actual values, numerical and analytical results of the right ascension, declination and spin velocity and the pointing deviation are shown in Figs. 8, 9, 10. The deviation between the computed values and actual values of the right ascension, declination and spin velocity are shown in Figs. 11, 12, 13. The discontinuities 


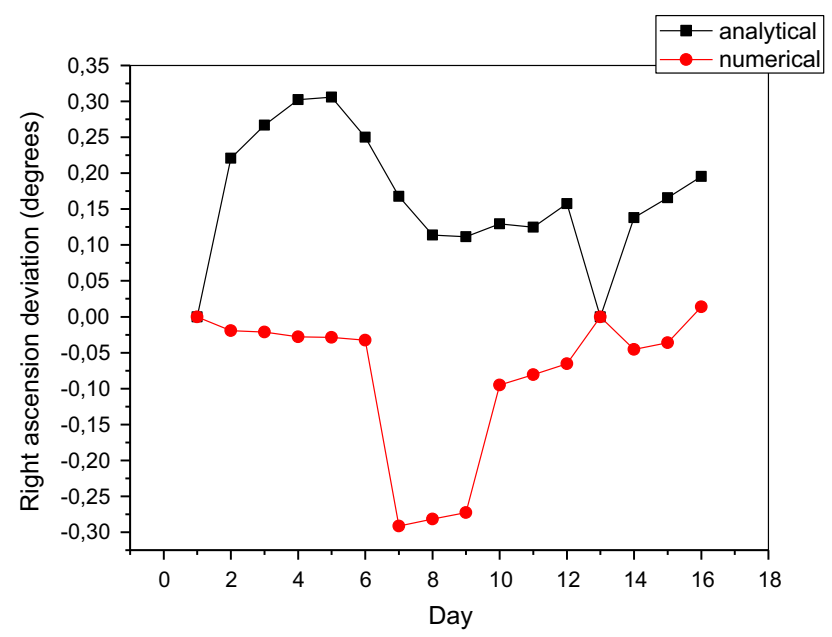

Fig. 11 Temporal variation for the deviation between the analytical or numerical and actual right ascension of the spin axis for SCD2, with daily updated data

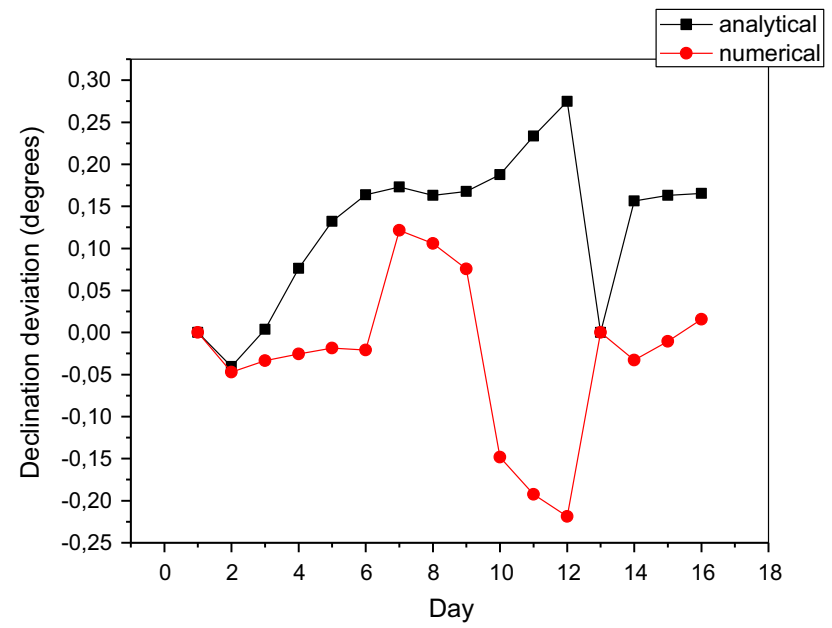

Fig. 12 Temporal variation for the deviation between the analytical or numerical and actual declination of the spin axis for SCD2, with daily updated data

in these figures occur due to the attitude control corrections applied by SCC/INPE. In Table 2 are shown the mean deviation errors for this simulation. It is important to note that when the attitude control actuates, the computed values are assumed to be equal to the real data because the control system is not included in the proposed theory.

For the test period of 16 days, the mean deviation errors are also within the dispersion range of the attitude determination system of SCC/INPE. In Table 2 it is possible to observe that all mean deviation errors in the numerical approach are smaller than these errors for analytical approach. 


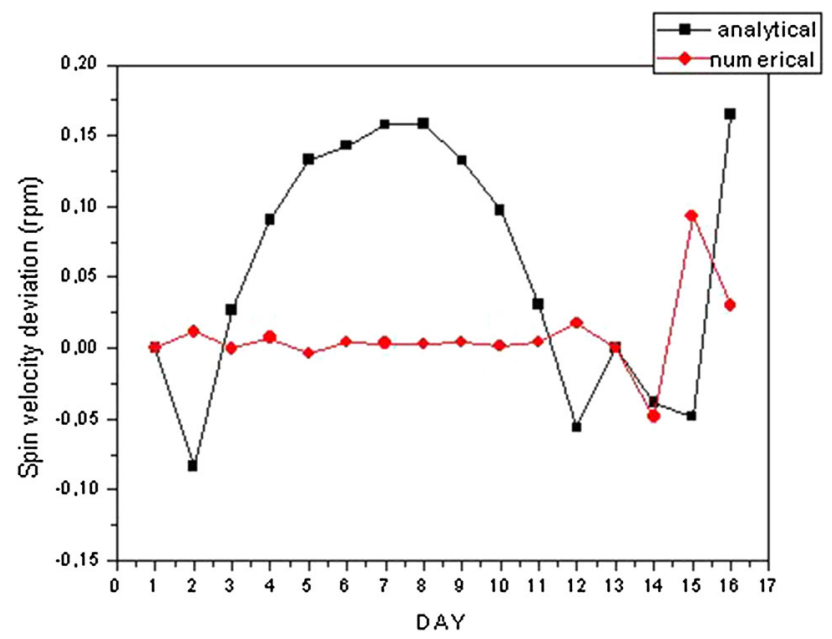

Fig. 13 Temporal variation for the deviation between the analytical or numerical and actual spin velocity for SCD2, with daily updated data

Table 2 Mean deviation errors for SCD2 simulations with daily updated data

\begin{tabular}{llc}
\hline & Numerical & Analytical \\
\hline Right ascension deviation (degrees) & -0.0819 & 0.1675 \\
Declination deviation (degrees) & -0.0269 & 0.1263 \\
Spin velocity deviation (rpm) & -0.008 & 0.0736 \\
\hline
\end{tabular}

\section{Conclusions}

In this paper, numerical and analytical approaches were presented to the spin-stabilized satellite attitude propagation taking into account the residual, eddy current, aerodynamic, solar radiation and gravity gradient torques. The numerical approach is developed in terms of the quaternion parametrization and the analytical approach in terms of the spin velocity, right ascension and declination of the spin axis.

Numerical simulations were presented to the spin-stabilized Brazilian's satellites SCD1 and SCD2, where the attitude and orbital data are daily updated with real attitude data supplied by INPE. The results show a good agreement between the computed and actual data during the simulated time interval. For both satellites, all the mean deviations are within the dispersion range of the attitude determination system required for these satellites.

In general, the mean deviation errors of the numerical approach are smaller than the analytical approach. The results are better than the others Zanardi et al. 2014, because the numerical approach was updated.

The results show that the average components of the external torque are sufficient to propagate the spacecraft attitude. It is also important to observe that the time simulation for the analytical is faster than numerical approach.

The results for the exponencial decay for the spin velocity and the precession of the spin axis agree with the results of Kuga et al. (1987a, 1999) and Yu (1963). 
Acknowledgments This present work was supported by FAPESP, proc. 2012/21023-6, and by CAPES/PNVS.

\section{References}

Carrara V, Guedes UTVG (1994) Attitude control aspects for SCD1 and SCD2. RBCM-J Braz Soc Mechanical Sci 16:421-425

Garcia RV, Zanardi MC, Kuga HK (2009) Spin-stabilized spacecrafts: analytical attitude propagation using magnetic torques. Math Prob Eng 2009:1-19

Kuga HK, Silva WCC, Guedes UTV (1987a) Attitude dynamic of th e spin stabilized satellite. São Jose dos Campos SP, Tecnichal Report, INPE-4403-NTE/275. INPE ( in Portuguese)

Kuga HK, Ferreira LD, Guedes UTV (1987b) Simulação de atitude e de manobras para o satélite brasileiro estabilizado por rotação. Tecnichal Report, INPE-4271-PRE/1143 ( in Portuguese)

Kuga HK, Orlando V, Lopes RVF (1999) Flight dynamics operations during leop for the INPE's second environmental data collecting satellite SCD2. RBCM-J Braz Soc Mechanical Sci 21:339-344

Mitchell JW, Richardson DL (2001) A simplied kinetic element formulation for the rotation of a perturbed mass-asymetric rigid body. Celest Mech Dyn Astronom 81(1-2):13-25

Motta GB, Zanardi MC (2014) Environmental torques and the solar radiation. Proceeding of the 24th International Symposium on Space Flight Dynamics. Laurel, Maryland, USA, pp 1-18

Orlando V, Lopes RVF, Kuga HK (1997) INPE'S flight dynamics team experience thought four years of scd1 in orbit operations: main issues, improvements and tends. ESA International Symposium on Spaceflight Dynamics. Darmstadt, Alemanha, pp 433-437

Pisacane VL, Moore RC (1994) Fundamentals of Space System. Oxford University Press, New York

Shrivastava SK, Modi VJ (1993) Satellite attitude dynamics and control in the presence of enviromental torques: a brief survey. J Guidance Control Dyn 6(6):461-471

Thomas LC, Cappelare JO (1964) Attitude determination and prediction of spin stabilized satellites. Bell Syst Tech J 43(42):1654-1672

Wertz JR (1978) Spacecraft Attitude Determination and Control. D. Reidel, Dordrecht, Holanda

Yu EY (1963) Spin decay, spin-precession damping, and spin-axis drift of the telstar satellite. Bell Syst Tech J 42:2169-2193

Zanardi MC, Vilhena de Moraes R (1999) Analytical and semi-analytical propagation of artificial an satellite's rotational motion. Celest Mech Dyn Astronom 75:227-250

Zanardi MC, Orlando V, Bento RSP, Silva MF (2012) Satellites attitude propagation with quaternions. J Aerosp Eng Sci Appl 4(4):42-51

Zanardi MC, Orlando V, Motta GB, Cabette RE (2014) Numerical and analytical spacecraft attitude prediction. Proceedings of the 24th International Symposium on Space Flight Dynamics. Laurel, Maryland, USA, pp 1-18 